OPEN ACCESS

Edited by:

Astrid Müller,

Hannover Medical School, Germany

Reviewed by:

Özgür Albayrak,

Hannover Medical School, Germany Kate Tchanturia,

King's College London, UK

*Correspondence: Fernando Fernández-Aranda ffernandez@bellvitgehospital.cat

${ }^{+}$Shared first authorship.

Specialty section:

This article was submitted to

Eating Behavior,

a section of the journal

Frontiers in Psychology

Received: 15 October 2015

Accepted: 12 January 2016

Published: 02 February 2016

Citation:

Wolz I, Hilker I, Granero R, Jiménez-Murcia S, Gearhardt AN,

Dieguez C, Casanueva FF,

Crujeiras AB, Menchón JM and Fernández-Aranda F (2016)

"Food Addiction" in Patients with Eating Disorders is Associated with Negative Urgency and Difficulties to Focus on Long-Term Goals. Front. Psychol. 7:61. doi: 10.3389/fpsyg.2016.00061

\section{"Food Addiction" in Patients with Eating Disorders is Associated with Negative Urgency and Difficulties to Focus on Long-Term Goals}

\author{
Ines Wolz ${ }^{1,2+}$, Ines Hilker ${ }^{1 \dagger}$, Roser Granero ${ }^{2,3}$, Susana Jiménez-Murcia ${ }^{1,2,4}$, \\ Ashley N. Gearhardt' ${ }^{5}$, Carlos Dieguez ${ }^{2,6}$, Felipe F. Casanueva2,7, Ana B. Crujeiras ${ }^{2,7}$, \\ José M. Menchón ${ }^{1,3,8}$ and Fernando Fernández-Aranda ${ }^{1,2,4 *}$ \\ ${ }^{1}$ Department of Psychiatry, University Hospital of Bellvitge-IDIBELL, Barcelona, Spain, ${ }^{2}$ Ciber Fisiopatologia Obesidad y \\ Nutrición, Instituto Salud Carlos III, Barcelona, Spain, ${ }^{3}$ Department of Psychobiology and Methodology, Universitat \\ Autònoma de Barcelona, Barcelona, Spain, ${ }^{4}$ Department of Clinical Sciences, School of Medicine, University of Barcelona, \\ Barcelona, Spain, ${ }^{5}$ Department of Psychology, University of Michigan, Ann Arbor, MI, USA, ${ }^{6}$ Department of Physiology, \\ Centro Singular de Investigación en Medicina Molecular y Enfermedades Crónicas, University of Santiago de \\ Compostela-Instituto de Investigación Sanitaria, Santiago de Compostela, Spain, ${ }^{7}$ Laboratory of Molecular and Cellular \\ Endocrinology, Research Area, Complejo Hospitalario Universitario de Santiago de Compostela, A Coruña, Spain, ${ }^{8}$ Ciber \\ Salud Mental, Instituto Salud Carlos III, Barcelona, Spain
}

Objectives: The present study aimed to investigate if eating disorder patients differ in specific personality traits depending on a positive screening of food addiction (FA) and to find a model to predict FA in eating disorder patients using measures of personality and impulsivity.

Methods: Two hundred seventy eight patients, having an eating disorder, self-reported on FA, impulsivity, personality, eating and general psychopathology. Patients were then split into two groups, depending on a positive or negative result on the FA screening. Analysis of variance was used to compare means between the two groups. Stepwise binary logistic regression was used to obtain a predictive model for the presence of FA.

Results: Patients with FA had lower self-directedness, and more negative urgency and lack of perseverance than patients not reporting addictive eating. The probability of FA can be predicted by high negative urgency, high reward dependence, and low lack of premeditation.

Conclusion: Eating disorder patients who have more problems to pursue tasks to the end and to focus on long-term goals seem to be more likely to develop addictive eating patterns.

Keywords: eating disorder, food addiction, personality, impulsivity, negative urgency

Abbreviations: AN, anorexia nervosa; ANOVA, analysis of variance; BED, binge eating disorder; BN, bulimia nervosa; DSM, Diagnostic and Statistical Manual of Mental Disorders; ED, eating disorder; FA, food addiction; OSFED, other specified feeding or eating disorders; TCI, temperament and character inventory; YFAS, Yale Food Addiction Scale. 


\section{INTRODUCTION}

Until now there is no clear agreement about the question if FA is a valid and necessary concept, specifically in the domain of EDs. On the one hand, different components of food have been studied using animal models, providing evidence that sugar consumption - and to some extend also high fat food - can lead to addictive behaviors, similar to other substances of abuse (Teegarden and Bale, 2007; Avena et al., 2008, 2012; Gold and Avena, 2013). Hyperpalatable foods, characterized by high levels of sugar, fat and salt are potentially addictive for humans (Gearhardt et al., 2011a; Davis, 2014; Schulte et al., 2015). Apart from this, neuroimaging techniques have shed light over neural correlates of FA, as well as on the similarities between substance dependence and addictive-like eating behavior in humans in terms of reward value and incentive value of respective stimuli (Gearhardt et al., 2011b; Volkow et al., 2012; Davis et al., 2013; Smith and Robbins, 2013; Imperatori et al., 2014). On the other hand, the FA construct seems to overlap with common eating psychopathology, namely binging, and seems to have collinearity with severity of disordered eating. Furthermore, a much debated question is whether addictive properties intrinsic to specific foods (physical dependence) or rather the eating behavior per se (psychological dependence) play a major role in the explanation of addictive-like eating, wherefore the term "eating addiction" has been proposed in order to underline the behavioral component of these symptoms (see Hebebrand et al., 2014 for a review). This shows the need for more research on psychological processes underlying FA.

The Yale Food Addiction Scale (YFAS) was developed in 2009 with the aim to apply the diagnostic criteria for substance dependence of the fourth revision of the Diagnostic and Statistical Manual of Mental Disorders (DSM; American Psychiatric Association, 2013) to eating behavior (Gearhardt et al., 2009a). Since the development of this first validated tool for the measurement of addictive behaviors toward food, the number of publications about FA has experienced a constant growth (Gearhardt et al., 2011a). In DSM-5, the chapter on addictions has undergone reorganization, including now not only substance related disorders, but also behavioral addictions. FA could be included in this new category in future revisions of the DSM.

A meta-analysis including 23 studies using the YFAS reports a mean prevalence of FA of $19.9 \%$ in adult samples ranging from healthy normal weight, over obesity, to BED, and BN, in which the highest prevalence of up to $100 \%$ was found (Pursey et al., 2014). In a recent study using the YFAS in ED patients, $72.8 \%$ of the sample fulfilled the criteria for FA compared to $2.4 \%$ of healthy controls, those ED patients who report FA showing higher ED severity and more general psychopathology (Granero et al., 2014). If ED patients with and without FA differ on basic psychological measures, such as personality and impulsivity traits, focused approaches to treatment may be helpful. However, there is a lack of literature analyzing personality vulnerabilities underlying FA.

The idea, that personality characteristics implicated in addictive processes could also contribute to ED, is not a new concept and has been confirmed by empiric data (Davis and
Claridge, 1998; Lent and Swencionis, 2012). ED patients are more likely than healthy controls to use addictive substances such as tobacco, but also illicit drugs (Krug et al., 2008), which supports the notion of an "addictive personality." Yet, it is possible that this association is explained by those patients fulfilling the criteria of FA, rather than being typical to all ED patients. Assuming that FA is comparable to other (substance and/or behavioral) addictions, it is expectable that, after controlling for ED subtypes, patients having a positive FA screening will have more addictive-like personality traits than those who do not fulfill the YFAS criteria for FA.

A recent meta-analysis on temperament in ED (Atiye et al., 2015) shows high harm avoidance in all ED-types compared to controls, high novelty seeking in BN patients, high persistence in $\mathrm{AN}, \mathrm{BN}$ and Other Not Specified Eating or Feeding Disorders (OSFED), and no differences in reward dependence between patient and control groups. Furthermore, all types of ED-patients were found to have lower scores in self-directedness than healthy controls (Fassino et al., 2004). By comparison, the personality profile found in individuals with substance related and non-substance related addictive disorders, namely gambling disorder, shows similarities but also differences: high novelty seeking and low self-directedness was reported transdiagnostically for different drugs (Le Bon et al., 2004; Pedrero Pérez and Rojo Mota, 2008) and nonsubstance related addictions (Alvarez-Moya et al., 2007), harm avoidance in contrast may vary depending on the substance consumed (Schneider et al., 2015) and on sex (Clinton et al., 2004; Claes et al., 2012a; Granero et al., 2014). When comparing behavioral addictions (gambling disorder, compulsive buying) to $\mathrm{BN}$, high novelty seeking is more specifically related to the former group, whereas low self-directedness is associated to both groups and reward dependence is not clearly related to either of the groups (Alvarez-Moya et al., 2007; Jiménez-Murcia et al., 2015). Harm avoidance in general is high in both clinical groups, but may be a more gender specific trait, with lower values in males than in females (Alvarez-Moya et al., 2007; Claes et al., 2012a).

Since impulsivity is an important characteristic common to behavioral and substance addictions (Lawrence et al., 2009; Alvarez-Moya et al., 2011; Kaiser et al., 2012; Jiménez-Murcia et al., 2013; Ochoa et al., 2013; Torres et al., 2013; Di Nicola et al., 2015), heightened levels could also be associated with FA. However, high impulsivity has also been found in ED patients (Davies et al., 2009; Claes et al., 2012b, 2015), wherefore a clarification is needed of whether this correlate is related to ED in general, or if it relates specifically to addictivelike eating. In studies using different self-report measures (UPPS, Barratt Impulsivity Scale) in student populations, high impulsivity was related to higher scores on the YFAS (Davis et al., 2011); more specifically, negative urgency, lack of perseverance (Murphy et al., 2014; Pivarunas and Conner, 2015) and attentional impulsivity (Meule et al., 2012; Raymond and Lovell, 2015), while motor and non-planning impulsivity were related to FA only in one (Raymond and Lovell, 2015) of these studies. Regarding behavioral response inhibition 
TABLE 1 | Demographic and selected clinical data for the sample.

\begin{tabular}{|c|c|c|c|c|c|c|c|c|c|c|c|c|c|}
\hline & \multicolumn{2}{|c|}{ Total $n=278$} & \multicolumn{2}{|c|}{ AN $n=68$} & \multicolumn{2}{|c|}{$\mathrm{BN} n=110$} & \multicolumn{2}{|c|}{ OSFED $n=61$} & \multicolumn{2}{|c|}{ BED $n=39$} & $x^{2}$ & $d f$ & $p$ \\
\hline Gender females & \multicolumn{2}{|c|}{$92.8 \%$} & \multicolumn{2}{|c|}{$89.7 \%$} & \multicolumn{2}{|c|}{$96.4 \%$} & \multicolumn{2}{|c|}{$95.1 \%$} & \multicolumn{2}{|c|}{$84.6 \%$} & 7.46 & 3 & 0.059 \\
\hline Employed & \multicolumn{2}{|c|}{$69.4 \%$} & \multicolumn{2}{|c|}{$58.8 \%$} & \multicolumn{2}{|c|}{$70.0 \%$} & \multicolumn{2}{|c|}{$78.7 \%$} & \multicolumn{2}{|c|}{$71.8 \%$} & 6.19 & 3 & 0.103 \\
\hline Tobacco use & \multicolumn{2}{|c|}{$30.6 \%$} & \multicolumn{2}{|c|}{$29.4 \%$} & \multicolumn{2}{|c|}{$31.8 \%$} & \multicolumn{2}{|c|}{$39.3 \%$} & \multicolumn{2}{|c|}{$15.4 \%$} & 6.57 & 3 & 0.087 \\
\hline Alcohol abuse & \multicolumn{2}{|c|}{$10.1 \%$} & \multicolumn{2}{|c|}{$7.4 \%$} & \multicolumn{2}{|c|}{$17.3 \%$} & \multicolumn{2}{|c|}{$6.6 \%$} & \multicolumn{2}{|c|}{$0 \%$} & 12.1 & 3 & 0.007 \\
\hline Other drugs use & \multicolumn{2}{|c|}{$12.9 \%$} & \multicolumn{2}{|c|}{$10.3 \%$} & \multicolumn{2}{|c|}{$19.1 \%$} & \multicolumn{2}{|c|}{$9.8 \%$} & \multicolumn{2}{|c|}{$5.1 \%$} & 6.75 & 3 & 0.080 \\
\hline \multirow[t]{2}{*}{ Food addiction: screening positive } & \multicolumn{2}{|c|}{$74.8 \%$} & \multicolumn{2}{|c|}{$55.9 \%$} & \multicolumn{2}{|c|}{$89.1 \%$} & \multicolumn{2}{|c|}{$62.3 \%$} & \multicolumn{2}{|c|}{$87.2 \%$} & 33.08 & 3 & $<0.001$ \\
\hline & Mean & $S D$ & Mean & $S D$ & Mean & $S D$ & Mean & $S D$ & Mean & $S D$ & $\boldsymbol{F}$ & $d f$ & $p$ \\
\hline Age (years-old) & 29.1 & 10.4 & 26.7 & 9.2 & 28.7 & 9.4 & 26.6 & 10.2 & 38.3 & 10.9 & 14.4 & $3 ; 274$ & $<0.001$ \\
\hline Age of onset (years-old) & 19.7 & 8.4 & 17.7 & 5.1 & 19.0 & 7.0 & 19.8 & 9.9 & 24.6 & 11.8 & 5.8 & $3 ; 274$ & 0.001 \\
\hline Duration of illness (years) & 9.3 & 9.0 & 8.3 & 9.1 & 9.8 & 8.7 & 6.9 & 6.9 & 13.6 & 11.1 & 4.6 & $3 ; 274$ & 0.004 \\
\hline $\mathrm{BMI}\left(\mathrm{kg} / \mathrm{m}^{2}\right)$ & 24.5 & 8.7 & 16.8 & 1.5 & 25.2 & 6.4 & 22.7 & 4.2 & 38.9 & 9.1 & 125.0 & $3 ; 274$ & $<0.001$ \\
\hline Food addiction: total criteria & 4.76 & 1.89 & 3.51 & 1.71 & 5.69 & 1.46 & 3.74 & 1.72 & 5.92 & 1.36 & 42.93 & $3 ; 274$ & $<0.001$ \\
\hline
\end{tabular}

AN, anorexia nervosa; BED, binge eating disorder; BMI, Body Mass Index (kg/ $\left.\mathrm{m}^{2}\right)$; BN, bulimia nervosa; OSFED, Other Not Specified Eating or Feeding Disorders; SD, standard deviation.

tasks, FA was not consistently related to task performance (Meule et al., 2012, 2014a). These results show that the term "impulsivity" has been referred to in different ways and with varying meanings, which may explain the discrepant results of self-report measures of impulsivity when compared to behavioral impulsivity tasks (Cyders and Coskunpinar, 2011; Meule et al., 2014a) and shows that a clear definition of this construct is needed. In the following, impulsivity will be defined according to a five factor-model (Cyders et al., 2007) incorporating the facets lack of premeditation, lack of perseverance, sensation seeking, positive urgency and negative urgency.

The objectives of the present study were (1) to investigate if ED patients differ in specific personality traits depending on a positive FA screening according to the YFAS; and (2) to find a model to predict FA in ED patients using measures of personality and impulsivity. More specifically, starting from the literature on addictive personality traits, it was hypothesized that ED patients with FA would have more novelty seeking, similar self-directedness, reward dependence and harm-avoidance (1a), and higher negative urgency and lower perseverance than ED patients without FA (1b). The second objective was more explorative; therefore, we did not make specific hypotheses on which variables would best predict FA.

\section{MATERIALS AND METHODS}

\section{Participants}

Participants ( $n=278,20$ males) were recruited from consecutive referrals to the ED Unit of the Department for Psychiatry of Bellvitge University Hospital during a period comprised from September 2013 until March 2015. AN $(n=68)$, $\mathrm{BN}(n=110)$, BED $(n=39)$, and OSFED $(n=61)$ patients were originally diagnosed according to DSM-IV-TR
(American Psychiatric Association, 2000) criteria by means of the Structured Clinical Interview for DSM Disorders-I (First et al., 1996), conducted by experienced psychologists and psychiatrists. DSM-IV diagnoses were reanalyzed post hoc using the recent DSM-5 criteria to ensure diagnoses reflected the current diagnostic criteria (American Psychiatric Association, 2013). See Table 1 for sociodemographic variables, for further information on the sample characteristics see Supplementary Tables S1 and S2.

\section{Assessment}

Yale Food Addiction Scale-Spanish Version -YFAS-S (Gearhardt et al., 2009b; Granero et al., 2014)

The YFAS measures FA using 25 items which are assigned to seven scales, referring to the seven criteria for substance dependence defined by the DSM-IV: (1) tolerance, (2) withdrawal, (3) substance taken in larger amount/period of time than intended, (4) persistent desire/unsuccessful efforts to cut down, (5) great deal of time spent to obtain substance, (6) important activities given up to obtain substance, (7) use continued despite psychological/physical problems (American Psychiatric Association, 2000). The YFAS was translated into Spanish and validated in the Spanish adult and ED population, with good validity and reliability scores (Granero et al., 2014).

For the following analyses, we either used the "FA total criteria," which indicates the number of fulfilled subscales, or the positive versus negative screening result. If at least three of the seven criteria are met for a period of the last 12 months and the person feels significantly impaired and/or suffers due to the described behavior, this is referred to as "positive YFAS screening score." Internal consistency for the YFAS in our sample was excellent, Cronbach's $\alpha=0.92$.

\section{UPPS-P Impulsive Behavior Scale-UPPS (Whiteside and Lynam, 2001; Cyders et al., 2007)}

The UPPS-P measures five facets of impulsive behavior through self-report on 59 items: positive and negative urgency (tendency 
to act rashly in response to positive mood or to distress), lack of perseverance (inability to remain focused on a task), lack of premeditation (tendency to act without thinking of the consequences of an act) and sensation seeking (tendency to seek out novel and thrilling experiences). The Spanish translation shows good reliability (Cronbach's $\alpha$ between 0.79 and 0.93 ) and external validity (Verdejo-García et al., 2010). Reliability as measured by Cronbach's $\alpha$ for the UPPS-P in the study sample ranged from very good (negative urgency $\alpha=0.83$ ) to excellent (positive urgency $\alpha=0.91$ ).

\section{Temperament and Character Inventory-Revised-TCI-R (Cloninger, 1994)}

The TCI- $\mathrm{R}$ is a 240 -item self-report questionnaire measuring personality on four temperament and three character dimensions. The temperament dimensions are harm avoidance (inhibited, passive vs. energetic, outgoing); novelty seeking (approach to signals of reward, impulsivity vs. uninquiring, reflective); reward dependence (sociable, socially dependent vs. tough-minded, socially insensitive) and persistence (perseverant, ambitious vs. inactive, erratic). Character covers self-directedness (responsible, goal-directed vs. insecure, inept); cooperativeness (helpful, empathic vs. hostile, aggressive) and self-transcendence (imaginative, unconventional vs. controlling, materialistic). The original questionnaire and the Spanish version of the revised questionnaire were validated and show good psychometric properties (Cloninger, 1994; Gutiérrez-Zotes et al., 2004). Internal consistency for the TCI-R in the study sample ranged from very good (novelty seeking $\alpha=0.80$ ) to excellent (harm avoidance $\alpha=0.91)$.

\section{Eating Disorders Inventory-2-EDI-2 (Garner et al., 1983)}

The EDI-2 is a 91-item self-report questionnaire that assesses characteristics of $\mathrm{AN}$ and $\mathrm{BN}$ on the dimensions drive for thinness, bulimia, body dissatisfaction, ineffectiveness, perfectionism, interpersonal distrust, interoceptive awareness, maturity fears, asceticism, impulse regulation and social insecurity. This scale has been validated in a Spanish population (Garner, 1998), obtaining a mean internal consistency of $\alpha=0.63$.

\section{Symptom Check-List 90-Revised-SCL-90-R (Derogatis, 1994)}

The SCL-90-R is a self-report questionnaire measuring psychological distress and psychopathology through 90 items. The items load on nine symptom dimensions: somatization, obsessive-compulsive, interpersonal sensitivity, depression, anxiety, hostility, phobic anxiety, paranoid ideation and psychoticism. The global score (Global Severity Index, GSI), is a widely used index of psychopathological distress. The SCL has been validated in a Spanish sample obtaining a mean internal consistency of $\alpha=0.75$ (Derogatis, 2002).

\section{Behavioral and Substance Addictions}

Gambling, kleptomania, stealing and buying behavior and the abuse of alcohol, the use of tobacco (smoking on an at least a daily basis) and drugs (lifetime use of any drug other than alcohol and tobacco) were assessed in a clinical interview conducted by psychologists and psychiatrists experienced in the field of addictive behaviors.

\section{Procedure}

This study was approved by the local ethics committee and was conducted according to the Declaration of Helsinki. After participants signed informed consent, they were evaluated and diagnosed at the ED Unit of the University Hospital of Bellvitge by experienced psychologists and psychiatrists, who conducted two semi-structured face-to-face interviews. The first interview provided information about current ED symptoms, antecedents and other psychopathological data of interest. The second interview comprised psychometrical assessment, and weight (assessment of body mass index and body composition) and eating monitoring (through daily reports completed at home on food intake, purges, and binges).

\section{Statistical Data Analyses}

Statistical analyses were conducted with SPSS20 for windows. Since age significantly differed between groups and ED subtype is known to influence the probability of FA (Granero et al., 2014), these two variables were entered as covariates. ANOVA, adjusted by participants' age and ED subtype, was used to compare the means of the seven TCI-R and the five UPPS-P subscales between participants classified into the two FA groups (positive and negative screening score).

Regarding missing data, statistical analyses were performed for subjects with complete information on each instrument (pair-wise procedure). The number of missing data was very low in this study: only data from one SCL-90R questionnaire were missing (for one patient in the YFAS-negative group), one TCI-R (also for one patient in the YFAS-negative group) and eight UPPS (two patients of YFAS-negative and six patients of YFAS-positive group).

Stepwise binary logistic regression was used to obtain a predictive model for the outcome presence of a "positive YFAS screening score" (more than three criteria fulfilled and clinical significance), considering three blocks: the first block included and fixed the participants' sex, age and diagnostic subtype, the second block automatically selected the TCI-R scales with a significant prediction on the dependent variable, and the third block selected the UPPS-P scales with significant contribution. The predictive capacity of each block was measured through the increase in the Nagelkerke's pseudo$R^{2}$ coefficient and the goodness-of-fit of the final model through the Hosmer and Lemeshow test (Hosmer et al., 2013). Due to the multiple statistical comparisons, Bonferroni-Finner correction was included to avoid the increase in Type-I errors. The measure of the effect size for mean and proportion comparisons was done through the $95 \%$ confidence interval of the parameters and the Cohen's- $d$ coefficient (moderate effect 
TABLE 2 | Differences on mean scores of personality traits and impulsivity for patients with or without food addiction: ANOVA adjusted by age and ED subtype.

\begin{tabular}{|c|c|c|c|c|c|c|c|c|c|c|c|}
\hline \multirow[b]{4}{*}{ TCl-R: Novelty seeking } & \multicolumn{4}{|c|}{ Adjusted means; $S D$} & \multicolumn{7}{|c|}{ ANOVA } \\
\hline & \multirow{2}{*}{\multicolumn{2}{|c|}{$\begin{array}{c}\text { FA = negative } \\
n=70\end{array}$}} & \multirow{2}{*}{\multicolumn{2}{|c|}{$\begin{array}{c}\text { FA = positive } \\
n=208\end{array}$}} & \multicolumn{2}{|c|}{ FA $\times$ ED } & \multicolumn{5}{|c|}{ (adjusted by age and ED subtype) } \\
\hline & & & & & $F_{d f=3 ; 275}$ & ${ }^{1} p$ & $F_{d f=1 ; 275}$ & ${ }^{1} p$ & $e^{e t a}{ }^{2}$ & MD & $|d|$ \\
\hline & 100.39 & 15.07 & 100.71 & 15.83 & 0.36 & 0.781 & 0.02 & .915 & 0.000 & 0.32 & 0.02 \\
\hline TCl-R: Harm avoidance & 112.89 & 19.54 & 119.91 & 21.08 & 1.33 & 0.266 & 5.24 & .080 & 0.019 & 7.02 & 0.35 \\
\hline TCI-R: Reward dependence & 99.40 & 16.89 & 101.78 & 15.62 & 0.23 & 0.876 & 0.99 & 0.562 & 0.004 & 2.38 & 0.15 \\
\hline TCl-R: Persistence & 105.90 & 18.37 & 106.24 & 22.68 & 0.42 & 0.739 & 0.01 & 0.915 & 0.000 & 0.34 & 0.02 \\
\hline TCl-R: Self-directedness & 125.32 & 21.63 & 115.37 & 20.46 & 0.59 & 0.622 & 11.17 & 0.007 & 0.040 & -9.95 & 0.47 \\
\hline TCl-R: Cooperativeness & 136.49 & 17.33 & 134.07 & 16.24 & 0.29 & 0.835 & 1.02 & 0.562 & 0.004 & -2.43 & 0.14 \\
\hline TCl-R: Self-Transcendence & 63.32 & 13.28 & 63.88 & 14.27 & 2.00 & 0.114 & 0.07 & 0.915 & 0.000 & 0.57 & 0.04 \\
\hline UPPS: Lack premeditation & 23.48 & 6.08 & 23.38 & 6.24 & 0.07 & 0.974 & 0.01 & 0.912 & 0.000 & -0.10 & 0.02 \\
\hline UPPS: Lack perseverance & 21.44 & 5.45 & 23.54 & 5.96 & 0.79 & 0.500 & 6.22 & 0.033 & 0.023 & 2.10 & 0.37 \\
\hline UPPS: Sensation seeking & 27.50 & 8.01 & 25.27 & 8.80 & 0.71 & 0.546 & 3.41 & 0.110 & 0.013 & -2.23 & 0.26 \\
\hline UPPS: Positive UR & 27.07 & 8.79 & 29.13 & 8.99 & 0.21 & 0.892 & 2.34 & 0.159 & 0.009 & 2.05 & 0.23 \\
\hline UPPS: Negative UR & 29.68 & 6.70 & 34.39 & 6.56 & 0.22 & 0.881 & 24.50 & $<0.001$ & 0.085 & 4.70 & $0.71^{*}$ \\
\hline
\end{tabular}

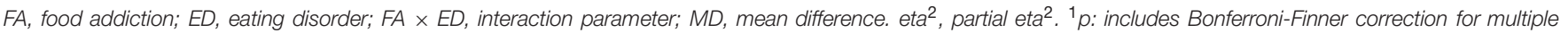
statistical comparisons. Bold: significant comparison (0.05 level). ${ }^{*}$ Bold: moderate $(|d|>0.50)$ to high $(|d|>0.80)$ effect size.

size was considered for $|d|>0.50$ and high effect size for $|d|$ $>0.80)$.

\section{RESULTS}

\section{Temperament, Character and Impulsivity Traits in ED Patients with and without Food Addiction}

Table 2 shows the results of the ANOVA comparing the temperament and character (TCI-R) and impulsivity (UPPS-P) traits mean scores between patients with positive versus negative YFAS screening score, adjusted by age and ED subtype. The analysis was carried out in two steps. In the first step the interaction parameter "positive YFAS screening score" by EDsubtype was included into the ANOVA to assess whether differences between individuals with positive and negative YFAS screening score were related to the different ED subtypes. Since this interaction term was not statistically significant, it was excluded from the model and the main effects of a "positive YFAS screening score" were estimated and interpreted. Results show that ED patients with positive FA screening compared to patients without FA have lower self-directedness $(p<0.01)$, while novelty seeking $(p=0.915)$, harm-avoidance $(p=0.08)$ and reward dependence $(p=0.56)$ do not differ significantly between groups. For a graphical representation and norm comparisons, see Supplementary Figure S1.

There were significant differences on the UPPS-P subscales lack of perseverance $(p<0.05)$ and negative urgency $(p<0.001)$, with higher values in FA patients compared to patients without "positive YFAS screening score" (see Table 2). Lack of premeditation, sensation seeking and positive urgency did not differ as a function of FA.

\section{Predictive Capacity of Personality in the Explanation of Food Addiction}

Table 3 includes the final predictive model of the presence of a positive YFAS screening score. The first block, including the covariates sex, age, and diagnostic subtype, obtained an initial predictive capacity equal to $R^{2}=0.22$. In the second block, the TCI-R reward-dependence and self-directedness scale scores were selected and fixed, with an increase in the predictive capacity equal to $R^{2}=0.08$, while the other TCI-R traits didn't explain further variance. In the third block, the UPPS-P lack of premeditation and negative urgency scores were included, and the new increase in the predictive ability was $R^{2}=0.08$, while the other UPPS-P subscales did not add additional explanative power. The final predictive model contained in the third block of the logistic regression indicates that after adjusting for sex, age, and ED subtype, the odds of a "positive YFAS screening score" is increased by high scores in the reward-dependence and negative urgency scales and low scores in the lack of premeditation scale, while negative urgency can be seen as the strongest predictor of FA. This model achieved goodness-of-fit (Hosmer-Lemeshow test: $p=0.408)$.

\section{DISCUSSION}

Our first goal was to determine if ED patients with FA differ in personality traits when compared with ED Patients without FA, after controlling for ED subtypes and age. Prevalence of FA is high in ED (Gearhardt et al., 2013; Granero et al., 2014; Meule et al., 2014b), in our sample $74.8 \%$ of participants met criteria for FA. Those with comorbid FA indeed showed a distinct personality profile, although it was different than expected from the literature regarding "addictive personality traits." FA was not related to higher values in novelty seeking, but exclusively 
TABLE 3 | Predictive model for the dependent variable: positive screening of food addiction.

\begin{tabular}{|c|c|c|c|c|c|c|c|c|}
\hline \multicolumn{2}{|c|}{ Criterion: FA "positive YFAS screening score" } & \multirow[t]{2}{*}{$B$} & \multirow[t]{2}{*}{$S E$} & \multirow[t]{2}{*}{ Wald } & \multirow[t]{2}{*}{$p$} & \multirow[t]{2}{*}{ OR } & \multicolumn{2}{|c|}{$95 \% \mathrm{Cl}(\mathrm{OR})$} \\
\hline \multicolumn{4}{|l|}{ First block $\left(\Delta R^{2}=0.221\right)$} & & & & & \\
\hline Sex $(0=$ female, $1=$ male $)$ & & -0.369 & 0.596 & 0.383 & 0.536 & 0.69 & 0.22 & 2.22 \\
\hline \multirow[t]{3}{*}{ Diagnostic subtype } & AN vs. OSFED & -0.293 & 0.398 & 0.541 & 0.462 & 0.75 & 0.34 & 1.63 \\
\hline & $B N$ vs. OSFED & 1.674 & 0.450 & 13.829 & $<0.001$ & 5.33 & 2.21 & 12.88 \\
\hline & $B E D$ vs. OSFED & 2.258 & 0.813 & 7.707 & 0.006 & 9.57 & 1.94 & 47.11 \\
\hline Sex $(0=$ female, $1=$ male $)$ & & -0.039 & 0.658 & 0.004 & 0.953 & 0.96 & 0.26 & 3.49 \\
\hline Age (years-old) & & 0.001 & 0.019 & 0.002 & 0.964 & 1.00 & 0.97 & 1.04 \\
\hline \multirow[t]{3}{*}{ Diagnostic subtype } & AN vs. OSFED & -0.011 & 0.420 & 0.001 & 0.978 & 0.99 & 0.43 & 2.25 \\
\hline & $B N$ vs. OSFED & 1.644 & 0.470 & 12.239 & $<0.001$ & 5.18 & 2.06 & 13.00 \\
\hline & $B E D$ vs. OSFED & 2.064 & 0.828 & 6.208 & 0.013 & 7.88 & 1.55 & 39.95 \\
\hline TCl-R: Reward-dependence & & 0.023 & 0.011 & 3.897 & 0.048 & 1.02 & 1.00 & 1.05 \\
\hline Age (years-old) & & 0.000 & 0.020 & 0.000 & 0.999 & 1.00 & 0.96 & 1.04 \\
\hline \multirow[t]{3}{*}{ Diagnostic subtype } & AN vs. OSFED & -0.048 & 0.452 & 0.011 & 0.916 & 0.95 & 0.39 & 2.31 \\
\hline & $B N$ vs. OSFED & 1.517 & 0.486 & 9.757 & 0.002 & 4.56 & 1.76 & 11.81 \\
\hline & $B E D$ vs. OSFED & 2.004 & 0.843 & 5.658 & 0.017 & 7.42 & 1.42 & 38.70 \\
\hline TCI-R: Reward-dependence & & 0.026 & 0.012 & 4.531 & 0.033 & 1.03 & 1.00 & 1.05 \\
\hline TCl-R: Self-directedness & & -0.016 & 0.011 & 2.209 & 0.137 & 0.98 & 0.96 & 1.01 \\
\hline UPPS: Lack premeditation & & -0.069 & 0.033 & 4.235 & 0.040 & 0.93 & 0.87 & 1.00 \\
\hline UPPS: Negative UR & & 0.124 & 0.034 & 12.992 & $<0.001$ & 1.13 & 1.06 & 1.21 \\
\hline Constant & & -2.439 & 2.358 & 1.070 & 0.301 & 0.09 & & \\
\hline
\end{tabular}

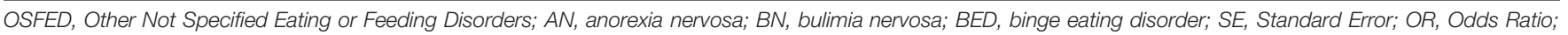
$\mathrm{Cl}$, Confidence Interval.

to lower self-directedness (1a). With regard to impulsivity, the hypothesis that ED patients with FA would have higher lack of perseverance and lower negative urgency was supported by our data (1b).

Lower self-directedness has been found to be a characteristic trait both in individuals with substance related and nonsubstance related addictive disorders, and seems to identify individuals more vulnerable to develop addictive behavior patterns (Alvarez-Moya et al., 2007; Schneider et al., 2015). In ED patients, low self-directedness is also a characteristic trait (Fassino et al., 2002; Cassin and Von Ranson, 2005; AlvarezMoya et al., 2007), but those with FA seem to be even more marked in this regard. Further support for our results is provided by another study (Bégin et al., 2012), that examined personality differences between overweight/obese women with and without FA and found that women with FA were more similar to women with substance use disorder than women without FA, particularly in regard to impulsivity and selfdirectedness.

Research has shown that harm avoidance is common to all ED subtypes and significantly higher in patients compared to controls (Cassin and Von Ranson, 2005; Lilenfeld et al., 2006; Atiye et al., 2015). In our study, both ED groups had values beyond the norms of general population (see Supplementary Figure S1), but no significant association was found between this temperament factor and a higher rate of FA. According to this data, we can thus infer that patients high in FA seem to have more problems with goal-orientation and accountability (as measured by self-directedness) compared to ED patients without FA, but both groups are comparable in behavioral and social inhibition and fear of uncertainty (as measured by harm-avoidance). Low self-directedness in patients high in FA implicates that this group has poor resourcefulness; this may present itself in problems to realistically adapt behavior to environmental requirements and to remain in accord with individual goals at the same time. Patients low in self-directedness may also be blaming and unreliable, which could lead to interpersonal problems in this patient group.

The results of this study further indicate that patients reporting addictive eating patterns have more difficulties to pursue tasks to the end and to focus on long-term goals, especially when they are in a negative mood. This is reflected by their high lack of perseverance and high values of negative urgency and is consistent with the results reported for nonclinical populations (Murphy et al., 2014; Pivarunas and Conner, 2015). It is interesting to note that FA patients show high impulsivity related to the regulation of negative emotions (as measured by negative urgency), but do not show elevated values in impulsivity related to positive emotions (as measured by positive urgency). Negative emotions may signal 
a discrepancy between personal needs and present conditions, which for individuals with high negative urgency is hard to bear (Cyders and Smith, 2008). This suggests that patients with FA feel a strong pressure to act immediately when having negative emotions instead of enduring until a moment more suitable to change. Since the need by itself all too often cannot be fulfilled immediately, ingestion of rewarding food can be seen as an attempt to escape these unbearable emotions by other means, which - depending on subjective expectancies - could also be a drug or another behavior (Fischer et al., 2012; Torres et al., 2013). Previous research shows that FA is also related to difficulties in emotion regulation (Gearhardt et al., 2012; Pivarunas and Conner, 2015), which corroborates the results on impulsive acts related to negative mood states.

Unexpectedly, ED patients with FA did not show elevated levels of novelty seeking when compared to ED patients without FA. In general, therefore, it seems that the approach to appetitive stimuli (reward seeking), which is implied by novelty/sensation seeking, does not differ between ED patients with and without addictive eating behavior. This points out that FA as assessed by the YFAS is more related to negative rather than to positive reinforcement, which is in line with results of a former study in normal weight participants (Meule and Kübler, 2012). It has been proposed that sensation seeking may be related rather to non-clinical drug use, than to an actual addiction (Torres et al., 2013), which would explain why patients with FA do not necessarily show elevated levels of sensation/novelty seeking.

In regard to the study's second objective, higher values in reward dependence, negative urgency and lack of premeditation and lower values in self-directedness together explained about $15 \%$ of the variance on having or not a positive FA screening, over and above sex, age, and diagnostic subtype, while negative urgency was the most important predictor and reduced the predictive power of the other variables to very small effects. Until now, risk factors for suffering FA have been established in different samples, e.g., students (Murphy et al., 2014; Pivarunas and Conner, 2015), obese women with overeating problems (Bégin et al., 2012) or in ED patients (Gearhardt et al., 2013; Granero et al., 2014; Meule et al., 2014b), but no study has explored which would be the highest risk population for presenting FA. Our prediction model suggests that individuals with a high disposition to act rashly to negative emotions are highly vulnerable for FA and would benefit from a specific approach for treating FA symptoms.

It is important to bear in mind the cross-sectional nature of our study; we cannot definitely conclude if the personality traits found to be related to FA precede or succeed FA symptoms, or if both have one common cause. Further work is required to confirm the interrelations between different predictors of FA in ED patients. Another limitation of this study is the small sample size, especially for male patients, wherefore results on effects of gender in FA should be investigated in future studies with higher sample power. Furthermore, our study only included one selfreport measure of FA, which could be completed by measures of craving, daily assessments and behavioral food ingestion tests in future studies.

Regarding the YFAS, a key issue is the high prevalence rates of FA in AN patients, which seems counterintuitive. Nevertheless, looking at the "total criteria fulfilled" (see Table 1), it appears that AN patients have a smaller number of total criteria fulfilled compared to $\mathrm{BN}$ and $\mathrm{BED}$; this may indicate to some part a problem of the cut-off criteria of the YFAS. In addition to this, our results show that the criteria most frequently fulfilled in AN patients are "important activities given up" (60.3\%) and "unable to cut down/stop" (89.7\%) (see Supplementary Table S3). Some of the items of the YFAS, such as those loading on "important activities given up" and "impairment or distress" may apply to $\mathrm{AN}$ in a similar way as to patients on the bulimic spectrum, wherefore this patient group also scores high on these criteria. On the other hand, the subscale "unable to cut down or stop" seems to be systematically misunderstood by AN patients, possibly due to their subjective feeling of eating too much. This could be addressed in future revisions of the scale and should be born in mind when employing the YFAS in this patient group.

It has been formerly suggested that FA may merely be an index of ED severity (Davis, 2013; Gearhardt et al., 2014). The data at hand suggests that ED patients with FA apart from showing a more severe symptomatology may differ from those without FA in the reward value they expect from food intake. Rather than enjoying the hedonic value of food in good mood, ED patients scoring high on FA mainly use food to regulate their negative emotions. It can be hypothesized that the relation between negative emotional states and food intake is mediated by impulsive personality traits and problems to focus on basic values or personal goals.

To improve the described emotional dysregulation and inhibition of responses, a training of emotion regulation strategies such as acceptance of emotional states could be helpful (Murakami et al., 2015). The importance to integrate work on emotions and emotion regulation skills into cognitive behavioral psychotherapy has reached increasing recognition in the last years (Kahl et al., 2012; Moyal et al., 2015), and new therapy approaches for ED patients have been developed. One example is the Cognitive Remediation and Emotion Skills Training (CREST), a manualized brief psychotherapy addressing emotion regulation and recognition (Money et al., 2011; Tchanturia et al., 2015), where patients learn to differentiate between different emotions and are taught about the communicative function of negative emotions. Patients with addictive-like eating patterns might benefit from this kind of training; the findings of our study further suggest that work on value-oriented behavior is important for patients with FA. Furthermore, this patient group might benefit to a great extend from learning to endure negative emotions by the use of strategies other than food intake and by this means they may be able to gradually reduce their dependence on food/eating in order to regulate negative mood states.

The psychological basis of addictive-like eating compared to mere ED, e.g., the importance ascribed to body shape, food-related cognitions, emotion regulation, should be further investigated in future studies. Which situations and emotional states lead to uncontrolled food intake in each group and the 
cognitions going along with this behavior could be investigated in experimental studies or ecological momentary assessment studies.

\section{AUTHOR CONTRIBUTIONS}

IW and IH contributed to the design of the work, acquisition and interpretation of the data. RG was responsible for statistical analysis and for writing statistical sections of the manuscript. SJ-M, AG contributed to administering and interpreting the psychological tests of this study. CD, FC, AC, JM, FF-A participated in the design of the study. All authors (IW, IH, RG, SJ-M, AG, CD, FC, AC, JM, FF-A) contributed to critically revising the work, approved the final version of the article to be published and agreed to be accountable for all aspects of the work in ensuring that questions related to the accuracy or integrity of any part of the work are appropriately investigated and resolved.

\section{REFERENCES}

Alvarez-Moya, E. M., Jiménez-Murcia, S., Granero, R., Vallejo, J., Krug, I., Bulik, C. M., et al. (2007). Comparison of personality risk factors in bulimia nervosa and pathological gambling. Compr. Psychiatry 48, 452-457. doi: 10.1016/j.comppsych.2007.03.008

Alvarez-Moya, E. M., Ochoa, C., Jiménez-Murcia, S., Aymamí, M. N., Gómez-Peña, M., Fernández-Aranda, F., et al. (2011). Effect of executive functioning, decision-making and self-reported impulsivity on the treatment outcome of pathologic gambling. J. Psychiatry Neurosci. 36, 165-175. doi: 10.1503/jpn.090095

American Psychiatric Association (2000). Diagnostic and Statistical Manual of Mental Disorders, 4th Edn. Washington, DC: American Psychiatric Association.

American Psychiatric Association (2013). Diagnostic and Statistical Manual of Mental Disorders, 5th Edn. Available at: dsm.psychiatryonline.org

Atiye, M., Miettunen, J., and Raevuori-Helkamaa, A. (2015). A meta-analysis of temperament in eating disorders. Eur. Eat. Disord. Rev. 23, 89-99. doi: 10.1002/erv.2342

Avena, N. M., Bocarsly, M. E., and Hoebel, B. G. (2012). Animal models of sugar and fat bingeing: relationship to food addiction and increased body weight. Methods Mol. Biol. 829, 351-365. doi: 10.1007/978-1-61779-458-2_23

Avena, N. M., Rada, P., and Hoebel, B. G. (2008). Evidence for sugar addiction: behavioral and neurochemical effects of intermittent, excessive sugar intake. Neurosci. Biobehav. Rev. 32, 20-39. doi: 10.1016/j.neubiorev.2007.04.019

Bégin, C., St-Louis, M.-E., Turmel, S., Tousignant, B., Marion, L.-P., Ferland, F., et al. (2012). Does food addiction distinguish a specific subgroup of overweight/obese overeating women? Health 4, 1492-1499. doi: $10.4236 /$ health.2012.412A214

Cassin, S. E., and Von Ranson, K. M. (2005). Personality and eating disorders: a decade in review. Clin. Psychol. Rev. 25, 895-916. doi: 10.1016/j.cpr.2005.04.012

Claes, L., Islam, M. A., Fagundo, A. B., Jimenez-Murcia, S., Granero, R., Agüera, Z., et al. (2015). The relationship between non-suicidal self-injury and the UPPS$\mathrm{P}$ impulsivity facets in eating disorders and healthy controls. PLoS ONE 10:e0126083. doi: 10.1371/journal.pone.0126083

Claes, L., Jimenez-Murcia, S., Agüera, Z., Sánchez, I., Santamaría, J., Granero, R., et al. (2012a). Eating disorders and pathological gambling in males: can they be differentiated by means of weight history and temperament and character traits? Eat. Disord. 20, 395-404. doi: 10.1080/10640266.2012.715517

Claes, L., Mitchell, J. E., and Vandereycken, W. (2012b). Out of control? Inhibition processes in eating disorders from a personality and cognitive perspective. Int. J. Eat. Disord. 45, 407-414. doi: 10.1002/eat.20966

Clinton, D., Björck, C., Sohlberg, S., and Norring, C. (2004). Patient satisfaction with treatment in eating disorders: cause for complacency or concern? Eur. Eat. Disord. Rev. 12, 240-246. doi: 10.1002/erv.582

\section{FUNDING}

Financial support was received from Fondo de Investigación Sanitaria -FIS (PI14/290) and co-funded by FEDER funds a way to build Europe. IW was supported by a predoctoral grant of AGAUR (2014FI_B 00372). CIBER Fisiopatología de la Obesidad y Nutrición (CIBERobn) and CIBER Salud Mental (CIBERsam), are both initiatives of INSTITUTO DE SALUD CARLOS III. The funders had no role in the study design, data collection and analysis, decision to publish, or preparation of the manuscript.

\section{SUPPLEMENTARY MATERIAL}

The Supplementary Material for this article can be found online at: http://journal.frontiersin.org/article/10.3389/fpsyg. 2016.00061

Cloninger, R. (1994). The Temperament and Character Inventory (TCI): A Guide to its Development and Use. St. Louis, MO: Center for Psychobiology of Personality.

Cyders, M., and Coskunpinar, A. (2011). Measurement of constructs using selfreport and behavioral lab tasks: is there overlap in nomothetic span and construct representation for impulsivity? Clin. Psychol. Rev. 31, 965-982. doi: 10.1016/j.cpr.2011.06.001

Cyders, M. A., and Smith, G. T. (2008). Emotion-based dispositions to rash action: positive and negative urgency. Psychol. Bull. 134, 807-828. doi: 10.1037/a0013341.Emotion-based

Cyders, M. A., Smith, G. T., Spillane, N. S., Fischer, S., Annus, A. M., and Peterson, C. (2007). Integration of impulsivity and positive mood to predict risky behavior: development and validation of a measure of positive urgency. Psychol. Assess. 19, 107-118. doi: 10.1037/1040-3590.19.1.107

Davies, H., Liao, P.-C., Campbell, I. C., and Tchanturia, K. (2009). Multidimensional self reports as a measure of characteristics in people with eating disorders. Eat. Weight Disord. 14, e84-e91. doi: 10.1007/BF033 27804

Davis, C. (2013). A narrative review of binge eating and addictive behaviors: shared associations with seasonality and personality factors. Front. psychiatry 4:183. doi: $10.3389 /$ fpsyt.2013.00183

Davis, C. (2014). Evolutionary and neuropsychological perspectives on addictive behaviors and addictive substances: relevance to the "food addiction" construct. Subst. Abuse Rehabil. 5, 129-137. doi: 10.2147/SAR.S56835

Davis, C., and Claridge, G. (1998). The eating disorders as addiction: a psychobiological perspective. Addict. Behav. 23, 463-475. doi: 10.1016/S03064603(98)00009-4

Davis, C., Curtis, C., Levitan, R. D., Carter, J. C., Kaplan, A. S., and Kennedy, J. L. (2011). Evidence that "food addiction" is a valid phenotype of obesity. Appetite 57, 711-717. doi: 10.1016/j.appet.2011.08.017

Davis, C., Loxton, N. J., Levitan, R. D., Kaplan, A. S., Carter, J. C., and Kennedy, J. L. (2013). "Food addiction" and its association with a dopaminergic multilocus genetic profile. Physiol. Behav. 118, 63-69. doi: 10.1016/j.physbeh.2013. 05.014

Derogatis, L. R. (1994). SCL-90-R Symptom Checklist-90-R. Administration, Scoring and Procedures Manual. Mineapolis, MN: National Computer System.

Derogatis, L. R. (2002). SCL-90-R. Cuestionario de 90 síntomas-Manual. Madrid: TEA Ediciones.

Di Nicola, M., Tedeschi, D., De Risio, L., Pettorruso, M., Martinotti, G., Ruggeri, F., et al. (2015). Co-occurrence of alcohol use disorder and behavioral addictions: relevance of impulsivity and craving. Drug Alcohol Depend. 148, 118-125. doi: 10.1016/j.drugalcdep.2014.12.028

Fassino, S., Abbate-Daga, G., Amianto, F., Leombruni, P., Boggio, S., and Rovera, G. G. (2002). Temperament and character profile of eating disorders: a 
controlled study with the temperament and character inventory. Int. J. Eat. Disord. 32, 412-425. doi: 10.1002/eat.10099

Fassino, S., Amianto, F., Gramaglia, C., Facchini, F., and Daga, G. A. (2004). Temperament and character in eating disorders: ten years of studies. Eat. Weight Disord. 9, 81-90. doi: 10.1007/BF03325050

First, M., Gibbon, M., Spitzer, R., and Williams, J. (1996). Users Guide for the Structural Clinical Interview for DSM-IV Axis I Disorders - Research Version (SCID-I, Version 2.0). New York, NY: New York State Psychiatric Institute.

Fischer, S., Settles, R., Collins, B., Gunn, R., and Smith, G. T. (2012). The role of negative urgency and expectancies in problem drinking and disordered eating: testing a model of comorbidity in pathological and at-risk samples. Psychol. Addict. Behav. 26, 112-123. doi: 10.1037/a0023460.The

Garner, D. M. (1998). Inventario de Trastornos de la Conducta Alimentaria (EDI2)-Manual. Madrid: TEA.

Garner, D. M., Olmstead, M. P., and Polivy, J. (1983). Development and validation of a multidimensional eating disorder inventory for anorexia nervosa and bulimia. Int. J. Eat. Disord. 2, 15-34. doi: 10.1002/1098108X(198321)2:2<15::AID-EAT2260020203>3.0.CO;2-6

Gearhardt, A., Corbin, W., and Brownell, K. (2009a). Food addiction: an examination of the diagnostic criteria for dependence. J. Addict. Med. 3, 1-7. doi: 10.1097/ADM.0b013e318193c993

Gearhardt, A. N., Corbin, W. R., and Brownell, K. D. (2009b). Preliminary validation of the Yale Food Addiction Scale. Appetite 52, 430-436. doi: 10.1016/j.appet.2008.12.003

Gearhardt, A., Davis, C., Kuschner, R., and Brownell, K. (2011a). The addiction potential of hyperpalatable foods. Curr. Drug Abuse Rev. 4, 140-145. doi: 10.2174/1874473711104030140

Gearhardt, A., Yokum, S., Orr, P., Stice, E., Corbin, W., and Brownell, K. (2011b). Neural correlates of food addiction. Arch. Gen. Psychiatry 68, 808-816. doi: 10.1001/archgenpsychiatry.2011.32

Gearhardt, A., White, M., Masheb, R., and Grilo, C. (2013). An examination of food addiction in a racially diverse sample of obese patients with binge eating disorder in primary care settings. Compr. Psychiatry 54, 500-505. doi: 10.1016/j.comppsych.2012.12.009

Gearhardt, A. N., Boswell, R. G., and White, M. A. (2014). The association of "food addiction" with disordered eating and body mass index. Eat. Behav. 15, 427-433. doi: 10.1016/j.eatbeh.2014.05.001

Gearhardt, A. N., White, M. A., Masheb, R. M., Morgan, P. T., Crosby, R. D., and Grilo, C. M. (2012). An examination of the food addiction construct in obese patients with binge eating disorder. Int. J. Eat. Disord. 45, 657-663. doi: 10.1002/eat.20957.An

Gold, M. S., and Avena, N. M. (2013). Animal models lead the way to further understanding food addiction as well as providing evidence that drugs used successfully in addictions can be successful in treating overeating. Biol. Psychiatry 74, e11. doi: 10.1016/j.biopsych.2013.04.022

Granero, R., Hilker, I., Agüera, Z., Jiménez-murcia, S., Sauchelli, S., Islam, M. A., et al. (2014). Food addiction in a Spanish sample of eating disorders: DSM-5 diagnostic subtype differentiation and validation data. Eur. Eat. Disord. Rev. 22, 389-396. doi: 10.1002/erv.2311

Gutiérrez-Zotes, J. A., Bayón, C., Montserrat, C., Valero, J., Labad, A., Cloninger, C. R., et al. (2004). [Temperament and Character Inventory Revised (TCI-R). Standardization and normative data in a general population sample]. Actas Esp. Psiquiatr 32, 8-15.

Hebebrand, J., Albayrak, Ö., Adan, R., Antel, J., Dieguez, C., De Jong, J., et al. (2014). "Eating addiction", rather than "food addiction", better captures addictive-like eating behavior. Neurosci. Biobehav. Rev. 47, 295-306. doi: 10.1016/j.neubiorev.2014.08.016

Hosmer, D. W., Lemeshow, S., and Sturdivant, R. X. (2013). Applied Logistic Regression, 3rd Edn. New York, NY: Wiley.

Imperatori, C., Innamorati, M., Contardi, A., Continisio, M., Tamburello, S., Lamis, D. A., et al. (2014). The association among food addiction, binge eating severity and psychopathology in obese and overweight patients attending low-energy-diet therapy. Compr. Psychiatry 55, 1358-1362. doi: 10.1016/j.comppsych.2014.04.023

Jiménez-Murcia, S., Granero, R., Moragas, L., Steiger, H., Israel, M., Aymamí, N., et al. (2015). Differences and similarities between bulimia nervosa, compulsive buying and gambling disorder. Eur. Eat. Disord. Rev. 23, 111-118. doi: $10.1002 /$ erv. 2340
Jiménez-Murcia, S., Granero, R., Stinchfield, R., Fernández-Aranda, F., Penelo, E., Savvidou, L. G., et al. (2013). Typologies of young pathological gamblers based on sociodemographic and clinical characteristics. Compr. Psychiatry 54, 1153-1160. doi: 10.1016/j.comppsych.2013.05.017

Kahl, K. G., Winter, L., and Schweiger, U. (2012). The third wave of cognitive behavioural therapies: what is new and what is effective? Curr. Opin. Psychiatry 25, 522-528. doi: 10.1097/YCO.0b013e328358e531

Kaiser, A. J., Milich, R., Lynam, D. R., and Charnigo, R. J. (2012). Negative urgency, distress tolerance, and substance abuse among college students. Addict. Behav. 37, 1075-1083. doi: 10.1016/j.addbeh.2012.04.017.Negative

Krug, I., Treasure, J., Anderluh, M., Bellodi, L., Cellini, E., Dibernardo, M., et al. (2008). Present and lifetime comorbidity of tobacco, alcohol and drug use in eating disorders: a European multicenter study. Drug Alcohol Depend. 97, 169-179. doi: 10.1016/j.drugalcdep.2008.04.015

Lawrence, A. J., Luty, J., Bogdan, N. A., Sahakian, B. J., and Clark, L. (2009). Problem gamblers share deficits in impulsive decision-making with alcohol-dependent individuals. Addiction 104, 1006-1015. doi: 10.1111/j.13600443.2009.02533.x

Le Bon, O., Basiaux, P., Streel, E., Tecco, J., Hanak, C., Hansenne, M., et al. (2004). Personality profile and drug of choice; a multivariate analysis using Cloninger's TCI on heroin addicts, alcoholics, and a random population group. Drug Alcohol Depend. 73, 175-182. doi: 10.1016/j.drugalcdep.2003.10.006

Lent, M. R., and Swencionis, C. (2012). Addictive personality and maladaptive eating behaviors in adults seeking bariatric surgery. Eat. Behav. 13, 67-70. doi: 10.1016/j.eatbeh.2011.10.006

Lilenfeld, L. R. R., Wonderlich, S., Riso, L. P., Crosby, R., and Mitchell, J. (2006). Eating disorders and personality: a methodological and empirical review. Clin. Psychol. Rev. 26, 299-320. doi: 10.1016/j.cpr.2005. 10.003

Meule, A., Lutz, A., Vögele, C., and Kübler, A. (2012). Women with elevated food addiction symptoms show accelerated reactions, but no impaired inhibitory control, in response to pictures of high-calorie food-cues. Eat. Behav. 13, 423-428. doi: 10.1016/j.eatbeh.2012.08.001

Meule, A., Lutz, A. P. C., Vögele, C., and Kübler, A. (2014a). Impulsive reactions to food-cues predict subsequent food craving. Eat. Behav. 15, 99-105. doi: 10.1016/j.eatbeh.2013.10.023

Meule, A., and Kübler, A. (2012). Food cravings in food addiction: the distinct role of positive reinforcement. Eat. Behav. 13, 252-255. doi: 10.1016/j.eatbeh.2012.02.001

Meule, A., von Rezori, V., and Blechert, J. (2014b). Food addiction and bulimia nervosa. Eur. Eat. Disord. Rev. 22, 331-337. doi: 10.1002/erv.2306

Money, C., Davies, H., and Tchanturia, K. (2011). A case study introducing cognitive remediation and emotion skills training for anorexia nervosa inpatient care. Clin. Case Stud. 10, 110-121. doi: 10.1177/1534650110 396545

Moyal, N., Cohen, N., Henik, A., and Anholt, G. E. (2015). Emotion regulation as a main mechanism of change in psychotherapy. Behav. Brain Sci. 38, e18. doi: 10.1017/S0140525X14000259

Murakami, H., Katsunuma, R., Oba, K., Terasawa, Y., Motomura, Y., Mishima, K., et al. (2015). Neural networks for mindfulness and emotion suppression. PLoS ONE 10:e0128005. doi: 10.1371/journal.pone.0128005

Murphy, C. M., Stojek, M. K., and Mackillop, J. (2014). Interrelationships among impulsive personality traits, food addiction, and body mass index. Appetite 73, 45-50. doi: 10.1016/j.appet.2013.10.008

Ochoa, C., Alvarez-Moya, E. M., Penelo, E., Aymami, M. N., Gómez-Peña, M., Fernández-Aranda, F., et al. (2013). Decision-making deficits in pathological gambling: the role of executive functions, explicit knowledge and impulsivity in relation to decisions made under ambiguity and risk. Am. J. Addict. 22, 492-499. doi: 10.1111/j.1521-0391.2013.12061.x

Pedrero Pérez, E. J., and Rojo Mota, G. (2008). Diferencias de personalidad entre adictos a sustancias y población general. Estudio con el TCI-R de casos clínicos con controles emparejados. Adicciones 20, 251-262.

Pivarunas, B., and Conner, B. T. (2015). Impulsivity and emotion dysregulation as predictors of food addiction. Eat. Behav. 19, 9-14. doi: 10.1016/j.eatbeh.2015.06.007

Pursey, K. M., Stanwell, P., Gearhardt, A. N., Collins, C. E., and Burrows, T. L. (2014). The prevalence of food addiction as assessed by the Yale Food Addiction Scale: a systematic review. Nutrients 6, 4552-4590. doi: 10.3390/nu6104552 
Raymond, K.-L., and Lovell, G. P. (2015). Food addiction symptomology, impulsivity, mood, and body mass index in people with type two diabetes. Appetite 95, 383-389. doi: 10.1016/j.appet.2015.07.030

Schneider, R., Ottoni, G. L., Carvalho, H. W., De, Elisabetsky, E., and Lara, D. R. (2015). Temperament and character traits associated with the use of alcohol, cannabis, cocaine, benzodiazepines, and hallucinogens: evidence from a large Brazilian web survey. Rev. Bras. Psiquiatr. 37, 31-39. doi: 10.1590/1516-44462014-1352

Schulte, E. M., Avena, N. M., and Gearhardt, A. N. (2015). Which foods may be addictive? The roles of processing, fat content, and glycemic load. PLoS ONE 10:e0117959. doi: 10.1371/journal.pone.0117959

Smith, D. G., and Robbins, T. W. (2013). The neurobiological underpinnings of obesity and binge eating: a rationale for adopting the food addiction model. Biol. Psychiatry 73, 804-810. doi: 10.1016/j.biopsych.2012.08.026

Tchanturia, K., Doris, E., Mountford, V., and Fleming, C. (2015). Cognitive Remediation and Emotion Skills Training (CREST) for anorexia nervosa in individual format: self-reported outcomes. BMC Psychiatry 15:53. doi: 10.1186/s12888-015-0434-9

Teegarden, S. L., and Bale, T. L. (2007). Decreases in dietary preference produce increased emotionality and risk for dietary relapse. Biol. Psychiatry 61, 10211029. doi: 10.1016/j.biopsych.2006.09.032

Torres, A., Catena, A., Megías, A., Maldonado, A., Cándido, A., Verdejo-García, A., et al. (2013). Emotional and non-emotional pathways to impulsive behavior and addiction. Front. Hum. Neurosci. 7:43. doi: 10.3389/fnhum.2013.00043

Verdejo-García, A., Lozano, Ó, Moya, M., Alcázar, M. Á, and Pérez-García, M. (2010). Psychometric properties of a spanish version of the UPPS - P impulsive behavior scale: reliability, validity and association with trait and cognitive impulsivity. J. Pers. Assess. 92, 70-77. doi: 10.1080/002238909033 82369

Volkow, N. D., Wang, G. J., Fowler, J. S., Tomasi, D., and Baler, R. (2012). Food and drug reward: overlapping circuits in human obesity and addiction. Curr. Top. Behav. Neurosci. 11, 1-24. doi: 10.1007/7854_2011_169

Whiteside, S. P., and Lynam, D. R. (2001). The five factor model and impulsivity: using a structural model of personality to understand impulsivity. Pers. Individ. Dif. 30, 669-689. doi: 10.1016/S0191-8869(00)0 0064-7

Conflict of Interest Statement: The authors declare that the research was conducted in the absence of any commercial or financial relationships that could be construed as a potential conflict of interest.

The reviewer Özgür Albayrak and handling Editor Astrid Müller declared their shared affiliation, and the handling Editor states that the process nevertheless met the standards of a fair and objective review.

Copyright (c) 2016 Wolz, Hilker, Granero, Jiménez-Murcia, Gearhardt, Dieguez, Casanueva, Crujeiras, Menchón and Fernández-Aranda. This is an open-access article distributed under the terms of the Creative Commons Attribution License (CC BY). The use, distribution or reproduction in other forums is permitted, provided the original author(s) or licensor are credited and that the original publication in this journal is cited, in accordance with accepted academic practice. No use, distribution or reproduction is permitted which does not comply with these terms. 